\title{
CIUDADANÍA Y POLÍTICAS PÚBLICAS: EL RETO DE UN GOBIERNO ABIERTO
}

\section{CITIZENSHIP AND PUBLIC POLICY: THE CHALLENGE OF OPEN GOVERNMENT}

Oscar Cortés Abad'

\section{RESUMEN}

Vivimos tiempos de cambio. La sociedad y el nuevo entorno derivado de la crisis económica y financiera global está dando lugar a nuevos sectores, cambios en las reglas de juego y nuevas pautas de actuación de los diversos actores. Lo público como espacio común en el que se desarrollan las dinámicas económicas y sociales no va a ser menos y se verá fuertemente afectado por estos cambios debiendo evolucionar a nuevas formas de hacer política y Administración más centradas en el ciudadano. La tecnología será a su vez palanca e instrumento facilitador de este cambio. El presente artículo pretende descubrir las claves de este proceso. Para ello, analizaremos los elementos distintivos de la nueva economía y la nueva sociedad que se vislumbran en estos inicios del siglo XXI. Posteriormente identificaremos los elementos específicos del contexto digital al que se enfrentan los diferentes actores. Descubriremos las nuevas claves para hacer política y Administración que den respuesta a estas necesidades y plantearemos un nuevo paradigma llamado Gobierno Abierto.

\section{ABSTRACT}

We live in times of change. Society and the new environment resulting from the global financial and economic crisis is leading to new sectors, changes in the rules and new patterns of action of the various actors. The public as a common space in which they develop economic and social dynamics not going to be less and will be strongly affected by these changes must evolve to new forms of politics and administration more citizen-centered. The technology will turn lever and instrument to facilitate this change. This article aims to discover the keys to

\footnotetext{
${ }^{1}$ Profesor del Centro Universitario Villanueva
} 
this process. To do this, we will analyze the distinctive elements of the new economy and the new society that are emerging at the dawn of the century. Then identify the specific elements of the digital context faced by different actors. Discover the new keys for policy and administration that respond to these needs and will raise a new paradigm called Open Government.

PALABRAS CLAVE: Crisis económica y financiera global / Dinámicas económicas y sociales / Tecnología / Gobierno / Economía

KEYWORDS: Economic crisis and global financial / economic and social dynamics / Technology / Government / Economics

\section{Políticas públicas y creación de valor}

El concepto de "producción" en lo público tiene sus particularidades. En comparación con el sector privado, el sector público ha sido catalogado tradicionalmente como un sector "no productivo", consumidor de recursos, sin un retorno tangible fácil de expresar en resultados.

Es fácil comprender por qué no se asocia lo público como una actividad creadora de valor: la imagen de creación de valor está vinculada a un proceso de transformación física que da lugar a productos terminados que se pueden vender en un mercado a clientes que pagan un precio por ellos.

En el sector público es más complejo visualizar los procesos de creación de valor producidos dentro de políticas públicas definidas por decisiones políticas, hitos de gestión o actos administrativos.

Analicemos algunos casos concretos:

- Servicios: se trata de transacciones en las cuales un usuario de un determinado servicio recibe una prestación, directamente por la organización REVISTA DO DIREITO UNISC, SANTA CRUZ DO SUL $\mathrm{N}^{\circ} 39 \mid$ p. 67 - 88| JAN-JUL 2013 
pública o indirectamente por empresas privadas que prestan el servicio en cualquier régimen de colaboración público-privada. En estos casos el valor público no está simplemente en la prestación del servicio, sino también en los beneficios colaterales. Por ejemplo, en el servicio de recogida de basuras también se crea valor al sensibilizar a los ciudadanos en que sean "limpios" o prevenir enfermedades.

- "Capacidad de respuesta": se trata de inversiones públicas destinadas a mantener una cierta capacidad que únicamente será utilizada cuando se produzcan determinados sucesos. Hablamos, por ejemplo, de las fuerzas armadas, la policía o las unidades de bomberos. En estos casos, el valor público llega mediante la "disuasión" o el disponer de suficientes medios y entrenamiento para el caso que pueda existir una catástrofe medioambiental.

- Autoridad y regulación: en este caso el sector público produce normativa y seguridad jurídica a través de la aplicación del principio de autoridad.

- Infraestructura social: como por ejemplo los programas redistributivos, en los que el valor público aparece de diversas formas: incrementando la cohesión social mediante el fortalecimiento de los segmentos sociales beneficiarios del programa; transmitiendo el mensaje a la sociedad de que hay un respaldo o "red de seguridad" en caso de que las cosas vayan mal; fomentando valores, etc.

Vemos que en la actividad pública se prestan servicios, se fomenta, se informa, se producen derechos y obligaciones, se sanciona, etc. a ciudadanos, empresas, grupos de interés que a su vez son usuarios, contribuyentes, administrados y votantes.

Son estos elementos de producción, más abstractos, intangibles y a largo plazo, los más valiosos de la producción pública. Mientras que en la empresas privada la medición de la rentabilidad económica es directa, lo público tiene por objeto la consecución de "outcomes" o impactos de desarrollo 
social (número de muertos por habitante, velocidad media en la ciudad, etc.) cuyo valor final no tiene un esquema racional "causa-efecto" y tiende erróneamente a confundirse con indicadores de la actividad desempeñada por la organización en cuestión ("outputs").

\section{La situación de partida: el triángulo asimétrico en el poder público}

Fue a lo largo del siglo XX cuando al calor del avance en el desarrollo institucional de los Estados se produjo el "contrato social" que ha venido regulando el reparto de poder en el ejercicio de las funciones públicas. De forma figurada podríamos asimilar dicho contrato con un triángulo de poder, asimétrico, en cuyos vértices se situarían respectivamente ciudadanos, políticos y burócratas.

Por un lado los ciudadanos, que ceden su poder democrático a organizaciones políticas, económicas o sociales que supuestamente les representan y son las que interactúan en el tablero de juego de las políticas públicas.

Por otro los políticos, encuadrados en partidos, que tienen otorgado el rol del diseño, de la generación de ideas para promover el bienestar social, de la intermediación ante intereses contrapuestos.

Por último los burócratas, responsables directos de la producción, que se encuadran en estructuras de poder centralizado focalizadas en el cumplimiento de los procedimientos.

Encontramos pues una situación en la que desde la ciudadanía se ha producido una doble delegación que ha acentuado la asimetría de poder y ha llevado a un progresivo empequeñecimiento del ciudadano como sujeto político. Situación ésta que, pudiendo resultar cómoda para una mayoría, ha desembocado en un alejamiento de la "res pública" de sus principales 
protagonistas, los ciudadanos, convirtiéndoles en titulares de derechos y obligaciones que en no pocas ocasiones caen sobre papel mojado, decisores cada cuatro años, usuarios de servicios públicos "cosificados" o súbditos indefensos.

En esta situación, el poder se acaba dirimiendo en una pugna entre políticos y funcionarios en la que el ciudadano queda impotente como mero observador, en el eslabón más débil de la cadena. ¿Es sostenible esta situación?, ¿Hasta cuándo continuará acentuándose esta anomalía democrática? Veamos a continuación que algo está cambiando .....

\section{El nuevo entorno socio-económico}

Durante los casi dos siglos que han transcurrido de actividad pública la relación entre políticos, Administración y ciudadanía se ha desarrollado en entornos marcados por la estabilidad, la previsibilidad y la rigidez de los modelos burocráticos. La interacción viene produciéndose de acuerdo a procedimientos muy estructurados en los que la prioridad está en cumplir con objetividad las garantías otorgadas a los ciudadanos-administrados. A pesar de los esfuerzos modernizadores intentados en las décadas de los 80 y 90 del siglo pasado el cambio real en el sector público ha sido insuficiente.

Llegamos a nuestros días y encontramos un entorno extremadamente convulso en sus diversas dimensiones económica, social, política 0 tecnológica. Veamos por qué.

\section{Vector social}

Se caracteriza por la evolución hacia una sociedad cada vez más formada, compleja e interdependiente, la llamada sociedad del conocimiento, que demanda más y mejores servicios. Plural y diversa, fragmentada y crecientemente heterogénea debido a cambios demográficos originados por flujos migratorios y disminución de natalidad. Creciente número de actores y 
grupos de interés con cambios en las posiciones relativas de poder y una cierta crisis de legitimidad de "lo público".

\section{Vector tecnológico}

El prodigioso desarrollo de la tecnología es uno de los factores más rupturistas y transgresores a los que se enfrentan las organizaciones en las próximas décadas. Las nuevas tecnologías de la información y comunicaciones resultan un factor clave en la mejora de la competitividad ofreciendo la oportunidad de una gran transformación y acercamiento en el funcionamiento de las mismas.

\section{Vector económico}

Viene marcado por la crisis económica y financiera, la creciente globalización de los mercados, la gran significación de los flujos migratorios, las transacciones de capital y bursátiles, o la moderación del gasto público derivada de la crisis fiscal. Este último aspecto es especialmente significativo: la elevada y creciente demanda en número, tipología y calidad de servicios públicos supone una exigencia extrema para las cuentas públicas, teniendo en cuenta las dificultades para incrementar aún más la recaudación impositiva y las necesidades en políticas sociales y redistributivas disparadas producto de la crisis.

\section{Vector político-institucional}

Caracterizado por una complejidad derivada del número de actores, su tipología, las relaciones entre ellos, los diferentes niveles competenciales

(poder relativo) y la velocidad en el cambio de los mismos. El proceso de descentralización se ve complementado en paralelo por la interdependencia apareciendo estructuras e instituciones con ámbito de actuación supranacional. Irrumpen en el escenario conflictos de intereses, rivalidades y reivindicaciones en materia de competencias y financiación. 


\section{El poder de las redes y su relevancia en el nuevo escenario}

En 2003 apareció en EEUU el concepto de web social o web 2.0 de la mano de Tim O'Reilly.

La web social se ha convertido en un fenómeno reciente. El número de aplicaciones ha crecido de forma exponencial: servicios para publicar, distribuir y compartir contenidos (blogs, Flickr, Youtube), crear conocimiento colectivo (Wikipedia), almacenar información (de.li.cious) o redes que permiten la interacción social (Facebook).

Se está conformando una nueva realidad social y empresarial asociada al concepto 2.0. Una nueva generación de "nativos digitales" (Marc Prensky, 2001), individuos que han crecido utilizando internet más frecuentemente incluso que la televisión, algo impensable hace algunos años.

Esta utilización es diversa, variando desde una actividad más pasiva (lectura de blogs, visionado de videos en YouTube o visitas a redes sociales), pasando por una participación activa (comentarios a contenidos ya creados) hasta llegar a una franja minoritaria que se anima a crear contenidos propios. Hoy es difícil encontrar personas que no estén en redes sociales o espacios de micro-blogging como Twitter en los que los usuarios interactúan y se relacionan en micro-comunidades de forma ágil y dinámica a través de mensajes breves de 160 caracteres.

Lo cierto es que en el siglo XXI el capital ya no es el único elemento sobre el que se sustenta el poder. Emerge con fuerza el poder de la información, el cual se sustenta en la democratización de la misma, es decir, en el hecho de estar disponible de forma instantánea y sin coste, a un solo click, para millones de ciudadanos que puedan tener interés en utilizarla. 
Gracias a este empoderamiento ganan protagonismo las estructuras en red: individuos que no tienen contacto físico pero que comparten un determinado interés y se organizan, sin jerarquías ni líderes, para actuar. Es más sencillo pues cohesionar esfuerzos y movilizar recursos masivamente reduciendo costes de transacción, información y coordinación.

La mera posibilidad de que los ciudadanos puedan organizarse al margen de gobiernos o instituciones tiene como consecuencia inmediata el debilitamiento de las estructuras de intermediación social siendo preciso habilitar nuevos cauces de expresión que sirvan a unas personas que demandan no sólo respuestas sino también en muchos casos una búsqueda conjunta de soluciones.

Vemos pues que estamos ante el contexto ideal para el desarrollo de una ciudadanía cívica que se implique en su destino como sociedad. Y es en el entorno de la tecnología donde surge una nueva filosofía de vida, en lo personal y lo profesional, una nueva ética, la "ética hacker" (Himanen 2001), basada en una actitud vital, en poner pasión e ilusión en tu trabajo, en la ilusión de participar en algo importante.

La ética hacker es la que ha permitido que gran parte del salto evolutivo asociado a las TIC haya tenido lugar. Su secreto está en interiorizar una serie de valores: la capacidad de pensar en términos de comunidad, de colaboración, la presencia transparente, honesta, abierta y genuina, escuchar, asumir el riesgo a equivocarse, obtener el máximo valor mediante la colaboración y retornarlo a la comunidad.

La ciudadanía 2.0 es pues una cuestión de valores. La Internet de las personas supone emancipación y un rol más activo del usuario al posibilitar a éste coproducir contenidos, colaborar y participar en redes dinámicas que crecen y se retroalimentan. Se trata de conectar personas facilitando un entorno abierto que estimule a cooperar y compartir.

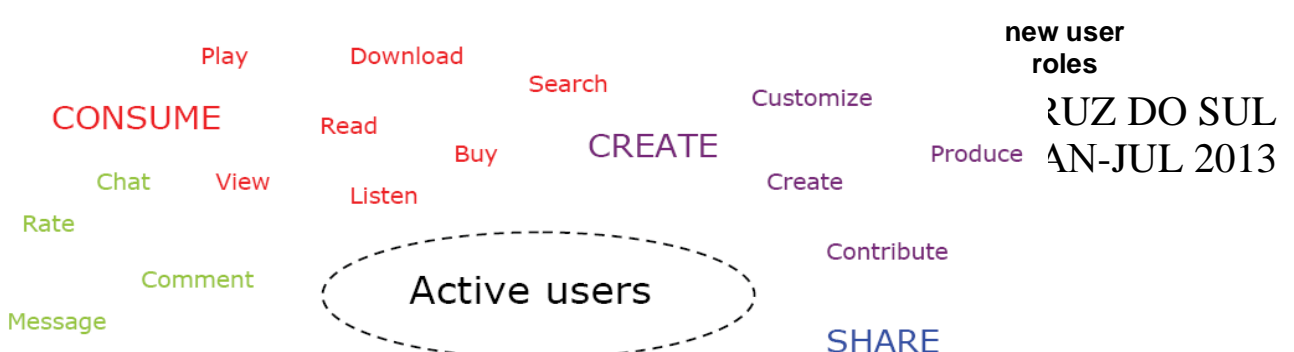


Fig 1.- Rol de los usuarios en la web 2.0 (Slot \& Frissen 2007)

Tres ejemplos bien distintos de comunidades virtuales más o menos organizadas pudieran ser: (i) la red de militantes y simpatizantes del Partido Demócrata norteamericano agrupados en la plataforma MoveOn.org desde la que generan opinión, agitan conciencias, recaudan fondos, promueven campañas, etc. (ii) la comunidad digital externa Patient Opinion en la que se recaban opiniones de los pacientes sobre distintos facultativos del sistema sanitario; (iii) comunidades de práctica más especializadas como Communities of Practice for Local Government donde una nube de profesionales o interesados en la modernización municipal expone sus casos, experiencias, etc.

Pero también hay aspectos controvertidos: las comunidades digitales no siempre sirven para reforzar la cohesión social. Hay voces que se alzan denunciando la posible "balcanización" del interés público al producirse una fragmentación del espacio social hacia "ghettos" aislados donde sus miembros tienden a enrocarse en actitudes más extremas. También está por comprobar la capacidad de que la energía social generada pueda encauzarse hacia la construcción de propuestas y alternativas sólidas que puedan institucionalizarse.

\section{Viejas estructuras, nuevos paradigmas}


No es posible acercarse a los nuevos medios mediante viejas estrategias.

Citando a Joan Prats, "la Administración sigue a la sociedad como la sombra al cuerpo" ; la cuestión es, ¿a qué distancia?. Sea cerca o lejos comprobamos cómo se abre un abanico de oportunidades para un reequilibrio en las relaciones sociedad - gobierno: la sociedad civil puede ganar poder para una mejor contribución en la creación de valor público y los gobiernos tienen la ocasión de ceder poder para ganar legitimidad.

La experiencia anima a ser cautos. Todavía hoy el modelo de gestión pública mayoritariamente vigente en nuestras Administraciones es el de la burocracia maquinal. Dicho modelo, que surge entre finales del siglo XIX y principios del XX a partir de las teorías de la división del trabajo de Taylor, se basa en la implementación de decisiones técnicas garantizando los principios de neutralidad, imparcialidad y objetividad, en la aplicación rigurosa de la normas y procedimientos. La Administración pública enfatiza su gestión a través de la producción de "actividad" y actúa de forma reactiva frente a las diferentes cuestiones sociales. El funcionario, con derecho a empleo vitalicio, supone la garantía de objetividad y de continuidad en el funcionamiento administrativo, por encima de los distintos vaivenes políticos.

Es después de la Segunda Guerra Mundial cuando en Europa se ponen en marcha los grandes servicios públicos- sanidad y educación - con la voluntad de universalizar el bienestar y redistribuir la riqueza entre todos los ciudadanos. Se trata de una construcción del Estado del Bienestar basada en la separación estricta en los ámbitos público y privado y el papel preeminente del Estado, autosuficiente y todopoderoso en la resolución de cuestiones de orden social y la prestación de servicios.

Pero el crecimiento del gasto público que lleva asociado este modelo requiere de una bonanza económica sostenible y un incremento en la presión fiscal, cuya propia legitimidad impide que sea ilimitado. Si a esto le añadimos 
las exigencias de estabilidad presupuestaria y las crecientes demandas de servicios públicos, se plantea la necesidad de evolucionar hacia un nuevo modelo de reparto de tareas entre Estado y Sociedad.

Producto de la llamada "crisis del Estado del Bienestar" y al calor de la implantación del modelo neoliberal en distingos países anglosajones (Reino Unido, Nueva Zelanda, etc.) surge la llamada Nueva Gestión Pública (NPM, New Public Management) como paradigma frontalmente opuesto al modelo urocrático.

La base de la NPM es la "empresarialización" de lo público mediante la aplicación directa de las técnicas de gestión privada a los distintos aspectos de la gestión. El individuo pasa de ser el "administrado" a ser "cliente". Frente a la neutralidad y el procedimiento, la NPM se orienta a resultados y a la flexibilidad; se introducen instrumentos de mercado (por ejemplo, competencia) y se procede a externalizar y a privatizar. El enfoque se desplaza desde la planificación y servicio hacia el coste y la eficiencia.

Pasados los años y de igual forma que existe una opinión casi unánime sobre la insuficiencia del modelo burocrático, se hace también cuestionable la eficacia de la NPM. Se le acusa de insensible hacia los valores intangibles muy importantes en el ámbito público-, irresponsable ante la demanda provocada por las necesidades sociales -por centrarse demasiado en los costes y la eficiencia-, arbitraria ante la pérdida del funcionario como elemento independiente o despreocupada hacia las minorías y el papel institucional.

Surge, como punto intermedio entre ambos modelos, un nuevo paradigma, el de la "gobernanza democrática", que apuesta por una Administración posibilitadora, confiada en una sociedad implicada y con valores de cohesión social, política y administrativa. Se caracteriza además por la cooperación público-privada, la gestión de redes y la interdependencia, combinando distintos modelos (jerárquico, mercado o red) según las 
circunstancias, la coproducción de servicios, responsabilidades compartidas, diálogo y lealtad entre los ámbitos público y privado.

No se trata de que las organizaciones privadas o de la sociedad civil tiendan a sustituir a lo público, sino de que cooperen y se complementen. La legitimidad del Estado no sólo se mantiene intacta, sino que se refuerza como garante del principio de legalidad y como elemento preeminente en la capacidad para asumir el liderazgo en la resolución de los problemas sociales, así como en garantizar la transparencia en la articulación de los ámbitos público-privados.

Vemos pues que el rol y organización que adopte el Estado debe ser coherente con el periodo histórico y con el entorno socio-económico que corresponda. En la actualidad la fuerza de las redes puede provocar que aquellos gobiernos que no entiendan la nueva forma de hacer política y se aferren a posiciones trasnochadas sean superados por el nuevo poder de una sociedad conectada y borrados del mapa político por los ciudadanos.

La respuesta desde lo público a esta sociedad en red variopinta necesariamente tiene que estar basada en un Estado multiforme, anclado en la propia sociedad. Y para ello es preciso evolucionar a nuevos modelos de gobierno más transparente, accesible y receptivo que satisfagan las crecientes demandas ciudadanas de información y servicios, preservando la seguridad y el cumplimiento de la ley.

\section{Gobierno abierto, la posible solución}

El Gobierno abierto (en inglés, Open Government) tiene su origen en los países anglosajones y escandinavos por la necesidad de adaptar la gestión pública a escenarios contemporáneos. Significa una nueva forma de gobernar acercando política e instituciones a los ciudadanos en sus diversas facetas de detección de necesidades, formulación de políticas, creación de valor en la producción o rendición de cuentas. Supone poner al ciudadano en el centro de la acción gubernamental, reforzando su papel de "accionista" de lo público, 
"usuario-receptor" de servicios y "co-productor" de valor público sea de forma directa o indirecta.

Una Administración que decide innovar y para ello decide transformarse en abierta es aquella que además de adoptar una nueva forma de relación con la ciudadanía, también lo hace con sus empleados, otras Administraciones y entidades colaboradoras. Conversa con ellos a fin de oír lo que dicen y solicitan, toma decisiones basadas en sus necesidades y preferencias, facilita la colaboración en el desarrollo de los servicios que presta y comunica todo lo que decide y hace de forma transparente. Es una Administración de doble dirección, escucha incluso más que habla.

El Open Government tiene un importante componente rupturista y transformador, empezando por el cambio de rol del ciudadano y lo que todo ello conlleva. Pero es también necesario tener en cuenta la lógica políticoadministrativa existente siendo erróneo pensar que es la solución a todas las disfunciones en la actividad pública. Sencillamente hay ámbitos donde no tiene sentido ni plantearse su aplicación.

Por tanto estamos hablando de un concepto de gran profundidad que demanda el desarrollo de una política pública concreta de naturaleza transversal. Una innovación sistémica que implica una redistribución de derechos y responsabilidades entre la sociedad, políticos y profesionales, una evolución hacia nuevas estructuras y tipos de organización

Costará por tanto décadas visualizar sus efectos. Por ello no vale con decir, hay que hacer: grandilocuentes declaraciones de principios en favor de un Gobierno/Administración abiertos sobran si no van acompañadas de una estrategia plasmada en objetivos, acciones, herramientas y presupuesto. Y desde luego, más allá de la simple utilización de una u otra aplicación de redes sociales. 
El modelo tomado de referencia desde el principio ha sido el de la Administración Obama (EEUU) que ya en su primer día de funcionamiento publicó allá por enero de 2009 publicó en su página web la "Declaración sobre Gobierno Abierto" detallando unos principios de actuación basados en tres pilares fundamentales: transparencia, participación y colaboración.

\subsection{Transparencia}

Un gobierno transparente fomenta y promueve la rendición de cuentas de la Administración ante la ciudadanía y proporciona información sobre lo que está realizando y sobre sus planes de actuación. El objetivo no es otro que poner en conocimiento de la ciudadanía toda la información necesaria para que pueda actuar de manera crítica.

Aunque últimamente se ha puesto de moda hablar de transparencia, no es algo nuevo. Fue allá por los años 70 cuando se empezaron a acuñar expresiones del tipo "abrir las ventanas del sector público" (Chapman y Hunt, 1987) para referirse a la necesidad de reducir la excesiva opacidad en el ejercicio del poder burocrático.

Durante décadas hablar de transparencia significó hablar de principios de acceso y libertad de información -FOI: freedom of information-, plasmados en normas cuyo objeto es proteger el "derecho a saber" permitiendo a los ciudadanos el acceso a datos en manos del Estado.

Pero ha sido con la llegada del siglo XXI cuando se han acentuado algunas tendencias de cambio que se venían anticipando. En este contexto, hablar de transparencia implica ir más allá de cumplir con un simple mandato legal o declaraciones simbólicas.

La transparencia hoy debe impregnar las decisiones en el marco de las políticas públicas, ser parte de un compromiso amplio y decidido con el gobierno abierto (Cortés, 2012). Supone además asumir que siendo el 
conocimiento uno de los elementos básicos en la capacidad de intervención de los actores en las políticas públicas una democracia de mayor calidad implica la universalización del mismo.

La transparencia real debe ir asociada a tres valores fundamentales: la accesibilidad - que la información sea fácilmente accesible a cualquier persona, en cualquier momento y en cualquier lugar; la receptividad - desde el gobierno y/o la Administración- y la equidad - para equilibrar el gap entre actores con mayor y menor capacidad de acceso.

Debe formar parte de las políticas públicas, modulando para cada política el grado de apertura, pero siempre teniendo en cuenta que de lo que se trata es de redistribuir el poder para que el proceso no acabe siendo frustrante y engañoso a ojos de los ciudadanos. Todo ello permite hablar de transparencia a diferentes niveles: el de información -unidireccional de las instituciones a los ciudadanos; el de consulta - integrando la voz ciudadana-; o el de delegación, cuando la ciudadanía se implica directamente en la valoración de políticas públicas (Ortiz de Zárate, 2012).

En cualquiera de los casos la transparencia debe ir más allá. Es preciso potenciar la utilización de las herramientas disponibles y crear espacios de contacto directo con la ciudadanía sustentados en plataformas para el debate informado y el trabajo común.

Pero se requiere también trabajar y educar en valores: por un lado en los principios de gestión para que la transparencia se asocie de forma inequívoca a la forma de gestionar los asuntos públicos; por otro porque tener instituciones transparentes es condición necesaria pero no suficiente, al precisar la existencia de un tejido cívico con capacidad y potencial para exigir y actuar en el campo de las políticas públicas. Ambos son aspectos que se retroalimentan: mayor transparencia redunda en mayor implicación cívica, que a su vez exige mayor transparencia. 


\subsection{Participación}

Un gobierno que promueve la participación favorece el derecho de la ciudadanía a participar activamente en la conformación de políticas públicas y anima a la Administración a beneficiarse del conocimiento y experiencia de los ciudadanos.

Un caso concreto son los gobiernos Wiki (wikigov) que ofrecen un modelo basado en los conceptos de código abierto y Wiki, permitiendo a los miembros de la comunidad el acceso directo a leyes, normas $u$ otro tipo de regulaciones a cuya formación pueden contribuir, por ejemplo, participando en su redacción. Cualquiera puede registrar una contribución, incluso anónimamente, y es preservada. Se trata de un proceso transparente, en el que toda deliberación se estructura en un plano de igualdad. Regulations.gov, es un ejemplo de wikipedia legislativa. Recoge todas las leyes que están siendo tramitadas y habilita a los ciudadanos a opinar sobre ellas y a proponer textos alternativos.

Estas herramientas pueden también servir para que los responsables públicos informen a los ciudadanos sobre el resultado de su gestión y recaben comentarios y opiniones de primera mano. Un ejemplo notable es el blog de José Antonio Rodríguez Salas, alcalde de Jun (un pequeño municipio de Granada, España), que nos muestra casi a diariamente con un formato variado (incluye fotos, videos, etc.) la actividad que desempeña en el pueblo a la par que los ciudadanos del mismo pueden opinar y comentar.

\subsection{Colaboración}

Un gobierno que promueve la colaboración implica y compromete a los ciudadanos y demás agentes en el propio trabajo de la Administración.

Lo puede hacer a partir de diversas iniciativas. Por ejemplo desde plataformas donde el Gobierno invita a innovar y a poner a disposición de la 
comunidad el resultado de dichas innovaciones. Promoviendo procesos de "crowdsourcing" o colaboración masiva en la que el gobierno se abre para que las decisiones que conciernen a todos sean tomadas en conjunto y aprovechen nuevas ideas que ciudadanía puede ofrecer. Una especie subcontratación social en la que se externalizan capacidades innovadoras existentes fuera del aparato administrativo; se trata de pasar de la sabiduría de los expertos tecnocracia- a la sabiduría de las masas.

Algunos casos concretos:

- Regulación: Peer-to-Patent es una plataforma web en la que se publican patentes que pueden ser probadas por expertos o por voluntarios en general. Las conclusiones son remitidas a la Oficina de Patentes y Marcas US (USPTO) para su evaluación y decisión.

- Colaboración transversal entre agencias: Intellipedia (intranet) es una plataforma wiki gestionada por la CIA, que permite la colaboración directa entre 14 agencias de inteligencia de US.

- Gestión del conocimiento: una asesoría jurídica internacional ha implantado una plataforma interna de gestión del conocimiento que permite compartir el conocimiento informal a través de blogs, grupos de noticias y etiquetado social. Aunque se trata de un caso del sector privado, podría ser aplicado fácilmente en el sector público.

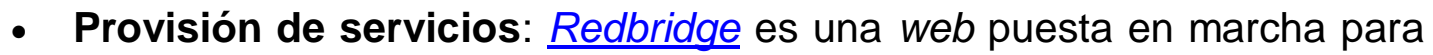
mejorar el Servicio Nacional de Salud. Recoge y publica las opiniones y valoraciones de los ciudadanos sobre el estado de los barrios como, cómo se podría gastar el dinero en ellos, etc.

- Cumplimiento de la ley: Mybikelane es una web en la que los ciclistas pueden publicar fotos de coches mal aparcados, junto con un comentario para dar a conocer el problema que desean denunciar.

\section{Las herramientas que posibilitan el gobierno abierto}


Nos encontramos en un momento interesante desde el punto de vista de aplicación de tecnologías al funcionamiento de gobiernos y Administraciones públicas en su relación con los ciudadanos.

La Administración electrónica es considerada uno de los núcleos fundamentales de la modernización administrativa. Consiste en la digitalización de servicios públicos incluyendo la relación virtual con los ciudadanos y la automatización de los trámites administrativos desarrollados en cada procedimiento: ventanillas, registros, firmas, expedientes, archivos, compulsas, etc. adquieren un nuevo apellido "electrónico" con el objeto de introducir criterios de eficiencia y calidad en el funcionamiento administrativo.

El nuevo replanteamiento del rol de la Administración consistente en el empowerment ciudadano coincide con el transfondo tecnológico y conceptual de la web 2.0 en una tendencia hacia una Administración abierta, cercana, flexible, receptiva e incentivadora de la innovación que nos lleva a hablar de Gobierno 2.0

Con el objeto de escuchar a los ciudadanos/empleados públicos, dotarles de cauces de participación, aprovechar la inteligencia colectiva, promover la colaboración y corresponsabilidad o mostrar los resultados de la gestión, existen numerosos ejemplos de utilización de herramientas web 2.0 (figura 2).

Fig.2: Herramientas web 2.0 en el sector público

\begin{tabular}{|l|l|}
\hline \multicolumn{1}{|c|}{ Tipo } & \multicolumn{1}{|c|}{ Descripción } \\
\hline Blogs & $\begin{array}{l}\text { Se comparten contenidos con los ciudadanos en un mismo } \\
\text { espacio bajo el formato elegido: vídeos, comentarios... } \\
\text { Información pública relevante distribuida a nuevas } \\
\text { audiencias. Dotar de rostro humano al gobierno usando un } \\
\text { tono informal. Apertura a conversaciones públicas. }\end{array}$ \\
\hline Wikis & $\begin{array}{l}\text { Liberando la información, por ejemplo, sobre una ciudad } \\
\text { para constituir una enciclopedia libre. Autoría y edición }\end{array}$ \\
\hline
\end{tabular}




\begin{tabular}{|c|c|}
\hline & $\begin{array}{l}\text { colaborativa, Grupos de trabajo o colaboración pública para } \\
\text { gestión de proyectos, conocimiento compartido, } \\
\text { aportaciones públicas. }\end{array}$ \\
\hline Vídeos & $\begin{array}{l}\text { Utilización de YouTube como canal de comunicación } \\
\text { amigable por la ciudadanía. Bibliotecas de vídeos, } \\
\text { formación y otras comunicaciones para audiencias on-line. }\end{array}$ \\
\hline Imágenes & $\begin{array}{l}\text { Fondo fotográfico con buscador consultable por año o } \\
\text { temática. Bibliotecas de fotos, nuevas audiencias. } \\
\text { Sensibilización y colaboración. }\end{array}$ \\
\hline Podcast & $\begin{array}{l}\text { Audio-guías orientadas a nuevos dispositivos como } \\
\text { reproductores mp3 que permiten total movilidad. Emisión } \\
\text { periódica de noticias y avisos, programas de radio } \\
\text { asíncronos, nuevas audiencias. }\end{array}$ \\
\hline $\begin{array}{l}\text { Redes } \\
\text { sociales }\end{array}$ & $\begin{array}{l}\text { Dinamización de comunidades. Conectar personas } \\
\text { globalmente, coordinación entre órganos y entre } \\
\text { administraciones. Gestión del conocimiento. Difusión de } \\
\text { eventos. }\end{array}$ \\
\hline Mashups & $\begin{array}{l}\text { Combinación de contenidos de múltiples fuentes para un } \\
\text { producto integrado, mejora del servicio, usabilidad y } \\
\text { funcionalidad del gobierno. Integración de datos externos. } \\
\text { Contenidos a disposición de otros creadores de mashups. }\end{array}$ \\
\hline $\begin{array}{l}\text { Micro- } \\
\text { blogging }\end{array}$ & $\begin{array}{l}\text { Forma de blogging que permite actualizaciones de textos } \\
\text { breves (tamaño de mensajes instantáneos), Consultas. } \\
\text { Mensajes: emergencias, noticias, etc; }\end{array}$ \\
\hline Widgets & $\begin{array}{l}\text { Permite la instalación del widget en una página web o blog } \\
\text { con información. Pequeñas aplicaciones y piezas de código } \\
\text { en páginas web o para uso de escritorio, aumentar el uso y } \\
\text { la usabilidad de las webs, informaciones y servicios } \\
\text { públicos. Llevar los contenidos a la home page del usuario. }\end{array}$ \\
\hline
\end{tabular}

\section{Conclusiones}

REVISTA DO DIREITO UNISC, SANTA CRUZ DO SUL $\mathrm{N}^{\circ} 39 \mid$ p. 67 - 88|JAN-JUL 2013 
Nos enfrentamos a un cambio sin precedentes en el ámbito socioeconómico provocado por nuevos desafíos en sus dimensiones humana, tecnológica o medioambiental. Este cambio afecta de manera directa a la conformación social distinguiéndose por la eclosión de un nuevo modelo de ciudadanía caracterizado por su heterogeneidad, nivel exigencia, preparación y utilización de las tecnologías. Frente a ello nos encontramos con una política y Administración diseñadas para afrontar los desafíos del siglo pasado, muy diferentes de los actuales.

La consecuencia inmediata es la necesidad de cambios en la forma de hacer política y Administración que provoquen un acercamiento entre los ciudadanos y sus instancias representativas. Cambios que van más allá de la simple aplicación de determinadas tecnologías afectando sin embargo a la forma de valorar y relacionarse con dicha sociedad. Cambios que, además, deben coexistir con la realidad presencial de una ciudadanía que mantiene todavía un marcado perfil analógico y con los esquemas burocráticos que marcan el funcionamiento de la realidad administrativa.

El gobierno abierto es condición necesaria pero no suficiente para buen gobierno. Una mayor apertura redundará en una mayor confianza en el gobierno, reforzará su legitimidad. Propiciará beneficios asociados a la inteligencia colaborativa, mejores resultados a menor coste. Los implicados cumplirán mejor con sus deberes porque participando la gente comprende y comprendiendo los niveles de cumplimiento son mayores. El umbral de acceso a decisiones se reducirá, aumentando la equidad y el estímulo a la innovación.

La introducción de paradigmas de esta naturaleza supone nuevas pautas para la actividad pública que deben encajar y coexistir amablemente con la realidad del ecosistema político-administrativo. Es preciso asumir que el cambio debe ser gradual, ganando apoyos y masa crítica, que no toda la actividad pública es fácilmente encajable en este nuevo modelo y que será preciso llevar a cabo estrategias claras a corto, medio, largo plazo que supongan valorar adecuadamente la dimensión de nuevos canales, centrarse 
en la transformación de servicios de utilidad real e incidir en la motivación y formación de empleados públicos.

\section{Referencias}

- Tim O'Reilly www.oreillynet.com

- Informe Anual sobre el Desarrollo de la Sociedad de la Información en España (Fundación Orange 2009)

- $\quad$ EIAA Mediascope Europe (2007)

- El tam-tam de los nativos digitales (El País, 21/12/2008)

- Blog de Genis Roca www.genisroca.com

- Kohut, A. The internet gains in politics, Pew Internet and American Life Project (2008)

- Wallsten K. (2005) Political blogs and the bloggers who blog them: is the political blogsphere and echo chamber?

- Blog de José Antonio Rodríguez Salas, alcalde de Jun http://www.granadablogs.com/joseantoniorodriguezsalas

- Blog Administraciones en red http://eadminblog.net

- Frissen V, Huijboom N., Kotterink, B. Towards a User Generated State? The impact of web 2.0 on government and the public sector, Paper for Media@Ise $5^{\text {th }}$ Anniversary Conference, (London, Sept 21-23)

- García E. Administración 2.0: Necesidades y retos por delante, Boletic

- Osimo D. Web 2.0 in Government: Why and How? European Commission Institute for Prospective Technological Studies (May 2008)

- Peer to patent www.peertopatent.org

- Redbridge www.redbridge.gov.uk

- Subirats, J. (2012): “¿Qué democracia y qué Administración para la nueva época? Internet y la gestión pública”. Ekonomiaz, 80, 2. cuatrimestre.

- Cortés, Ó. (2012): “La transparencia opaca”. Blog i-public@, 2 de abril. <http://i-publica.blogspot.com.es/2012/04/la-transparencia-opaca.html> 
- Ortiz de Zárate, A. (2012): Modelo LUDO: el gobierno abierto desde la perspectiva del ciclo de las políticas públicas. Grupo de Investigación en Gobierno, Administración y Políticas Públicas. Instituto Universitario de Investigación “Ortega y Gasset”. WP-2012-15. ISSN: 2174-9515.

- Chapman, R.; Hunt, M. (1987): Open Government. A Study of the Prospects of Open Government within the Limitations of the British Political System. Londres: Routledge.

- Himanen, P. (2001) La ética del hacker. Barcelona Destino

Professor Convidado 